\title{
Strategies for Improved Hospital Response to Mass Casualty Incidents
}

\author{
Mersedeh TariVerdi, PhD; Elise Miller-Hooks, PhD; Thomas Kirsch, MD, MPH, FACEP
}

\section{ABSTRACT}

Mass casualty incidents are a concern in many urban areas. A community's ability to cope with such events depends on the capacities and capabilities of its hospitals for handling a sudden surge in demand of patients with resource-intensive and specialized medical needs. This paper uses a whole-hospital simulation model to replicate medical staff, resources, and space for the purpose of investigating hospital responsiveness to mass casualty incidents. It provides details of probable demand patterns of different mass casualty incident types in terms of patient categories and arrival patterns, and accounts for related transient system behavior over the response period. Using the layout of a typical urban hospital, it investigates a hospital's capacity and capability to handle mass casualty incidents of various sizes with various characteristics, and assesses the effectiveness of designed demand management and capacityexpansion strategies. Average performance improvements gained through capacity-expansion strategies are quantified and best response actions are identified. Capacity-expansion strategies were found to have superadditive benefits when combined. In fact, an acceptable service level could be achieved by implementing only 2 to 3 of the 9 studied enhancement strategies. (Disaster Med Public Health Preparedness. 2018;12:778-790)

Key Words: capacity expansion strategies, discrete event simulation, emergency response, mass casualty incidents, surge capacity

\section{INTRODUCTION}

Mass casualty incidents (MCIs) have become of growing concern in many locations where the occurrence of natural or anthropogenic hazard events is rising. The Florida Department of Health ${ }^{1}$ categorizes MCIs by number of expected casualties. They specify categories I through $\mathrm{V}$ as having 5 through 10,11 to 20, 21 to 100,101 to 1000 , and $>1000$ casualties, respectively. Even smaller MCIs challenge a community's ability to provide quality patient care, quickly exhausting local rescue and response resources. MCIs may arise from more routine circumstances. For example, under severe weather conditions traffic accidents involving multiple vehicles and possible burn victims are common. Less probable but larger scale MCIs can arise due to human error, as would occur with the release of hazardous materials from a truck or train derailment. Larger MCIs may result from more catastrophic or less localized events, such as hurricanes. The US Federal Emergency Management Agency (FEMA) has identified specific disaster event types that might produce a MCI for which each state must be prepared.

Efficient response by the health care system, especially hospitals, is crucial to mitigating indirect loss of life and life-impacting injuries associated with MCIs. Preparedness steps and modified operational procedures designed for these types of incidents are needed for an efficient response, and are key elements in building resilient communities. Furthermore, techniques for quantifying the benefits of potential strategies for managing MCIs within the hospitals are warranted.

In a MCI, the health care system is faced with an abrupt increase in demand for resource-intensive care. The efficient management of critical resources in the form of staff (medical providers, nurses, etc.), stuff (oxygen, medical air, blood, hospital beds, etc.), and space (emergency rooms, operating rooms, laboratories, etc.) is crucial to meet surge demand requirements. Hick et $\mathrm{al}^{2}$ listed adaptive operational strategies for space and supply use under conventional, contingency, and crisis circumstances.

Only a few studies quantify hospital performance specific to a MCI. ${ }^{2-4}$ Of direct relevance is work by $\mathrm{Yi}$ et $\mathrm{al}^{3}{ }^{3}$ who simulate a generic hospital under varying demand-capacity ratios. A few additional works discuss the need for studying the operations of hospitals in MCIs, ${ }^{5,6}$ and others have conducted case studies to reveal the role of hospitals in close proximity to $\mathrm{MCI}$ locations for specific historical events. ${ }^{7}$ The literature has yet to tackle the complexities of hospital operations in a MCI. This paper seeks to address this gap. 
It is commonly assumed that waiting times for initial service will reach a steady state, after which point observations will reveal little new information. However, in the context of MCIs, nonstationary demand and service disruptions, among other demand and supply-side changes, produce transient time performance over the course of the response phase. Hospital system performance and its evolution over this period are greatly impacted by the initial system state: the number of available resources (eg, nurses, internal general ward [IGW] unoccupied beds, operating rooms [ORs]) and the number of patients waiting to receive services (eg, be admitted to the emergency department [ED], be admitted to an IGW, or be transferred to an OR). This research addresses system performance within the transient period under various initial states given dynamic demand and demand-responsive service capabilities. Few studies in any related field consider this transient system behavior. Those that do relate primarily to communications, supply chain management, and manufacturing., 8

In this paper, a patient-based, resource-constrained model of a generic urban hospital constructed in the Exendsim Simulation Software environment is described. A discrete event simulation modeling approach that uses an underlying queueing network conceptualization of the hospital's critical units was taken. The modeling approach extends the work that assesses a hospital operating in routine circumstances to $\mathrm{MCIs} .{ }^{10}$ This approach explicitly recognizes that the performance of the whole hospital depends on the functionality of its units and the specific needs and care paths of the patients. Since services share resources in hospitals, shortfalls in one unit can negatively affect services provided in another unit. Specific to a MCI are patient-flow dynamics, demand management strategies, operational strategies that are responsive to evolving demand patterns and time-varying service capacities, and capacity-expansion actions. These capacity-expansion, demand management, and operational strategies were designed based on in-depth interviews with key hospital personnel and author experience. ${ }^{11}$ These strategies include modified triage tactics, early-discharge decisions, speed-up in patient care procedures, and omission of some patient care services. In addition to presenting these strategies for efficient surge demand handling in a MCI, modeling adaptations for their replication are presented.

The interviews were conducted over multiple visits in the course of approximately one year at the Johns Hopkins Hospital, Suburban Hospital, and the Johns Hopkins Office of Critical Event Preparedness and Response. The primary sources (in addition to coauthors) included the director of operations and an administrative director of $\mathrm{ED} /$ trauma, safety, security and employee health services, among others. Extensive discussions regarding multiple hazard scenarios were held and findings were translated into modeling parameters.

Systematically designed experiments were conducted using this model to investigate the performance of the representative hospital under a range of $\mathrm{MCI}$ demand scenarios and response strategies. The experiments aimed to investigate (1) the functionality of a hospital and the impact of proposed capacity-expansion strategies (modifications to operations and alternative standards of care) in bottleneck formation in high-demand circumstances and (2) transient system behavior. The impacts of demand management through diversion of patients to alternative facilities and modified triage were also investigated.

\section{METHODS: HOSPITAL MCI MODELING}

The whole-hospital, resource-constrained model for level I and II trauma centers (hospitals) operating under a MCI scenario is described. Trauma level I hospitals are equipped at the highest level (of five levels) to provide total care for all injuries. The model builds on the framework developed in TariVerdi et $\mathrm{al}^{10}$ and thus differs not in its technical approach but rather in the details of the operations and patient flows. Modifications to routine operations and standards are proposed, and their potential for improved MCI handling is assessed.

\section{Patients}

In a MCI, patient arrival patterns depend on the circumstances of the event and unit service capacities depend on availability of required resources (staff and stuff). As patients arrive at the hospital, they are classified and prioritized based on injury type, severity, and prognosis. To handle a surge in demand for time-critical care, these triage protocols under a MCI differ from protocols implemented in routine circumstances. The outcome directly impacts the number of patients served and the burden on each of the care paths.

From a queuing perspective, within the arrival routine, customers arrive, each carrying individualized service requirements or survival likelihoods. Severity probability distributions typical of the MCI class determine individual patient survival likelihoods and therefore priorities. Survival likelihoods are a nonincreasing function of the time spent waiting for initial critical services based on patient injury category. If circumstances warrant, the lowest priority customers may be turned away without treatment. For customers who are accepted, customer priority, along with injury type, will dictate their assignment to specific care paths. Customers from classes with low priority are assumed to be impatient and may choose to renege if waiting times exceed a threshold.

For routine circumstances within the ED, the Emergency Severity Index (ESI) triage protocol ${ }^{12}$ is followed. An alternative prioritization scheme may be followed in a $\mathrm{MCI}$ when the number of patients entering the hospital in need of time-critical treatment exceeds a hospital's capabilities. Even before arrival at the hospital, while at the disaster scene, first responders triage the affected population using a rapid triage protocol, such as Simple Triage and Rapid Treatment (START). START classifies patients as having minor (green), delayed 
FIGURE 1

(A) Patient Classes at MCI Scene and Arrivals to Hospitals. (B) Dual Wave Phenomenon of MCI Patient Arrival Pattern. ${ }^{16}$ Abbreviations: EMS, emergency medical services; ESI, Emergency Severity Index; MCI, mass casualty incidents; START, Simple Triage Rapid Treatment.

(a)

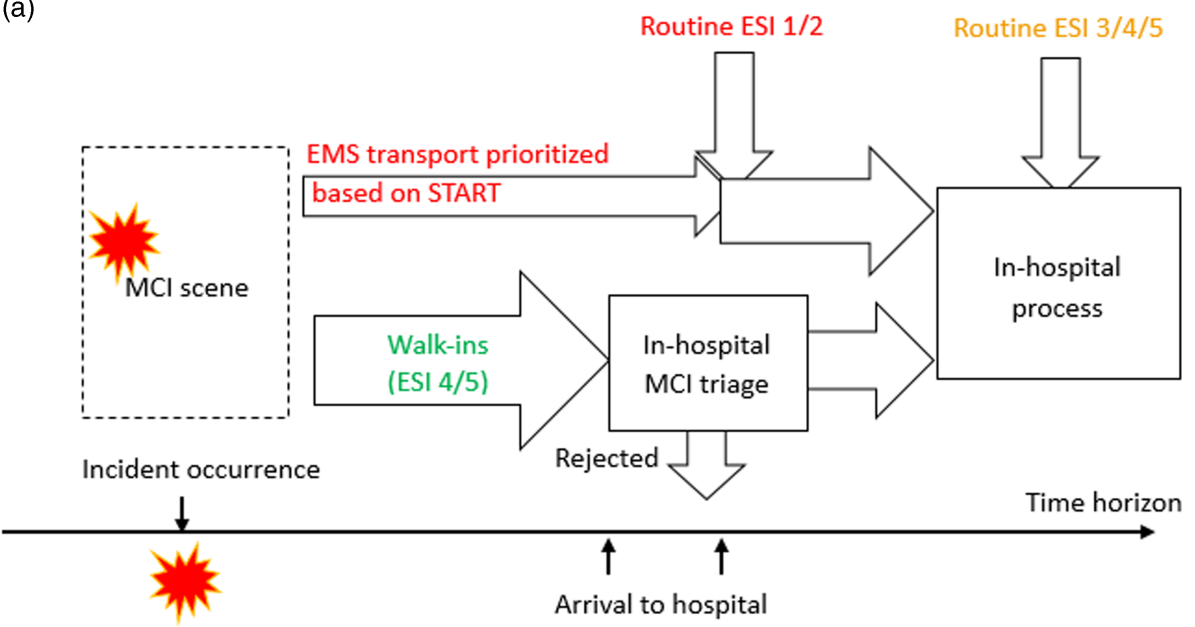

(b)

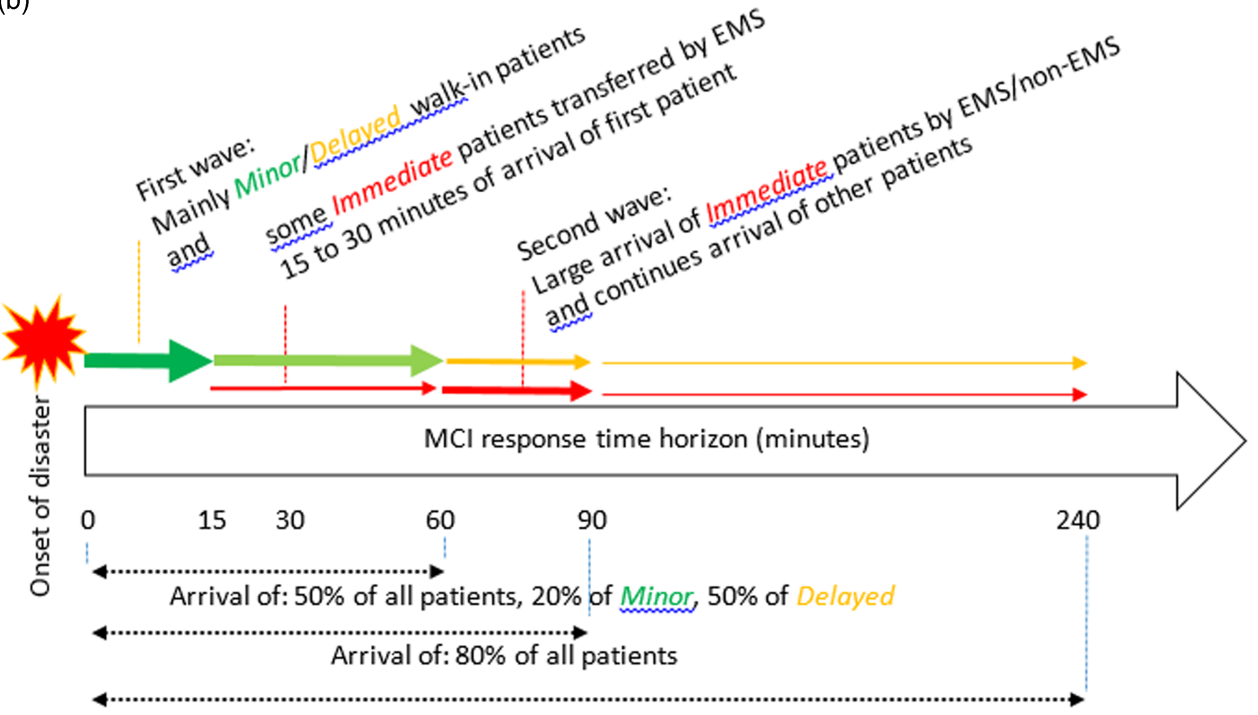

Arrival of: $95 \%$ of all patients

(yellow), immediate (red), and sometimes expectant (black; low survival likelihood and high resource needs). ${ }^{13}$ Following recommendations in the literature, ${ }^{14}$ maximum wait time thresholds are set to 1 to 1.5 hours for immediate patients and 6 to 8 hours for delayed patients. These categories determine how transportation resources at the incident scene are allocated and accelerate the in-hospital triage process (Figure 1A).

At the hospital, patients who arrive by ambulance or are tagged at the scene as immediate bypass the in-hospital triage process. Others, including those who walk in (accounting for approximately $90 \%$ of $\mathrm{MCI}$ patients), are categorized by injury type based on an Injury Severity Score (ISS) between 0 and 75. The ISS is computed based on affected body regions and severity levels. ISS and injury type determine each patient's survival likelihood, care path, and probable service times. Alternate classification methods for this purpose have been suggested in the MCI literature. ${ }^{3,15}$

In a $\mathrm{MCI}$, casualties can be estimated using disaster planning software, such as PACER or HAZUS-MH. As demand from the incident arises, a drop in routine patient arrivals by $25 \%$ during the response period can be expected ${ }^{17}$ and is assumed here. Some incident types, such as a pandemic, generate demand for the hospital that increases exponentially or linearly over time; whereas in other incident types, such as 
no-notice incidents, patient arrivals may increase rapidly but follow what is known as the "dual wave phenomenon" (Figure 1B). These arrival patterns (Figure 1B) are replicated within the arrival routine of the model. Batch arrivals replicate transport of groups of patients from the MCI scene. The patients in these groups are categorized as immediate or minor and arrive to hospital randomly following uniform distributions on intervals $[30,60]$ and $[15,30]$ minutes from incident start, respectively. Individual arrivals follow a nonstationary time-dependent Poisson distribution.

\section{Critical Hospital Services}

Within the queuing network, nodes represent locations of potential bottlenecks along critical service paths. These bottlenecks occur within hospital units but affect the flow of patients across units. Units included in the model, as shown in Figure 2B, are registration and triage; the ED, including fast-track and trauma units; operation theaters consisting of preoperation (preop) rooms, ORs, and postoperation rooms (postop), such as surgical intensive care units (SICUs), postanesthetic care units (PACUs), and stepdown units; intensive care units (ICUs); and IGWs. Laboratories and imaging, radiology, computed tomographic (CT) scan, and magnetic resonance imaging (MRI), are included in parallel to these units as part of diagnostic services. Specialized medical resources in terms of medical providers, nurses, technicians, and critical medical supplies are also explicitly modeled. For certain MCIs, it may be important to include decontamination units, burn units, or other specialized services. In this model, patient care paths are a function of patient medical needs and MCI-based operational strategies.

Federal guidelines ${ }^{16}$ dictate that health care facilities be selfsufficient for 48 to 96 hours in the aftermath of a MCI. During the crisis period, they must have the ability to reach a $30 \%$ increase in service capacity compared with routine operations. To expand the capacity within critical units during a MCI, a hospital may modify its operations or it may, in some circumstances, be able to lower the standards of care. For example, in a MCI registered nurses may be permitted to serve certain patients that doctors would ordinarily treat. Some standards of care are dictated to the hospital and can only be lowered under government directive (ie, state of emergency declaration). Modifications to operations thus serve two purposes: (1) to exploit untapped capacities and (2) to maximize service rates under required standards. Combinations of modifications and alternative standards are referred to herein as capacity-expansion strategies (Figure 2). Such strategies are proposed here and replicated within the models following assumptions described in Table 1.

The ED in routine ${ }^{10}$ and $\mathrm{MCI}$ conditions is depicted in Figures 2B and C. Changes to care paths of ESI level 4 and level 5 patients for MCI operations are noted.
Key differences between flows under routine and $\mathrm{MCI}$ models can be described with reference to admissions protocols, state-dependent care paths, and changes in service requirements as described in Appendix A. Additionally, information related to model outputs, complete list of monitored queues and patient exit points from the system in form of transfers and expirations (mortalities or hospital failure to stabilize patients in a timely manner) are included in Tables A1 and A2.

\section{Design of Numerical Experiments}

Numerical experiments were designed to assess the functionality of a representative, full-service hospital under MCI scenarios for varying demand scenarios under proposed capacity-expansion strategies. Strategies and demand scenarios are synopsized in Table 2.

Initial results were obtained to identify the number of runs required to achieve an acceptable error of 0.05 in expected waiting times. To achieve stability in run results while simultaneously accounting for rare events, 50 runs were conducted under each scenario. Each run replicated hospital operations over a multiday, postevent period, where the number of days considered is set to either 2 or 7 . While a 2-day period was sufficient to capture the operations immediately following a $\mathrm{MCI}, 7$ days were necessary for a pandemic scenario with multiday impact on demand. Each simulation run, thus, was made over 22 or 27 days, respectively, 20 of which were set as a simulation warm-up period. Data from the warm-up period were not used or reported. Figure 3 reflects this warm-up period for 7-day run results. Thirty-five combinations of modified operations (MOs) and alternative standards of care (ASC) as might be adopted were specified.

The inputs to the representative urban hospital model in terms of size (eg, number of beds, ORs), staffing, operational parameters, routing probabilities, and service times are summarized in Table 3.

The output from 50 modeled queues in the runs were monitored to evaluate hospital performance under combinations of demand inputs and capacity-expansion strategies. Average and maximum waiting times, number of patients in the queues, and queue lengths were collected for analysis. Commonly used metrics from routine operations may not be well-suited to MCI analyses. Instead, mortality and number of patients for which the hospital failed to provide timely service were computed. Because the physical condition of each patient changes over time and depends on services provided, individual probabilities of mortality change as the simulation progresses. Mortality was presumed from patient survival likelihoods given injury type and severity and whether the patients successfully received services in the simulation runs within a critical time period. 
FIGURE 2

(A) Hospital Patient Flow Model. ${ }^{10}$ (B) ED in Routine Conditions. ${ }^{10}$ (C) ED in MCI Conditions. Abbreviations: ED, emergency department; ESI, Emergency Severity Index; ICU, intensive care unit; IGW, inpatient general ward; ISS, Injury Severity Score; LWBS, leave without being seen; MCI, mass casualty incident; PACU, postanesthetic care unit; SICU, surgical intensive care unit.
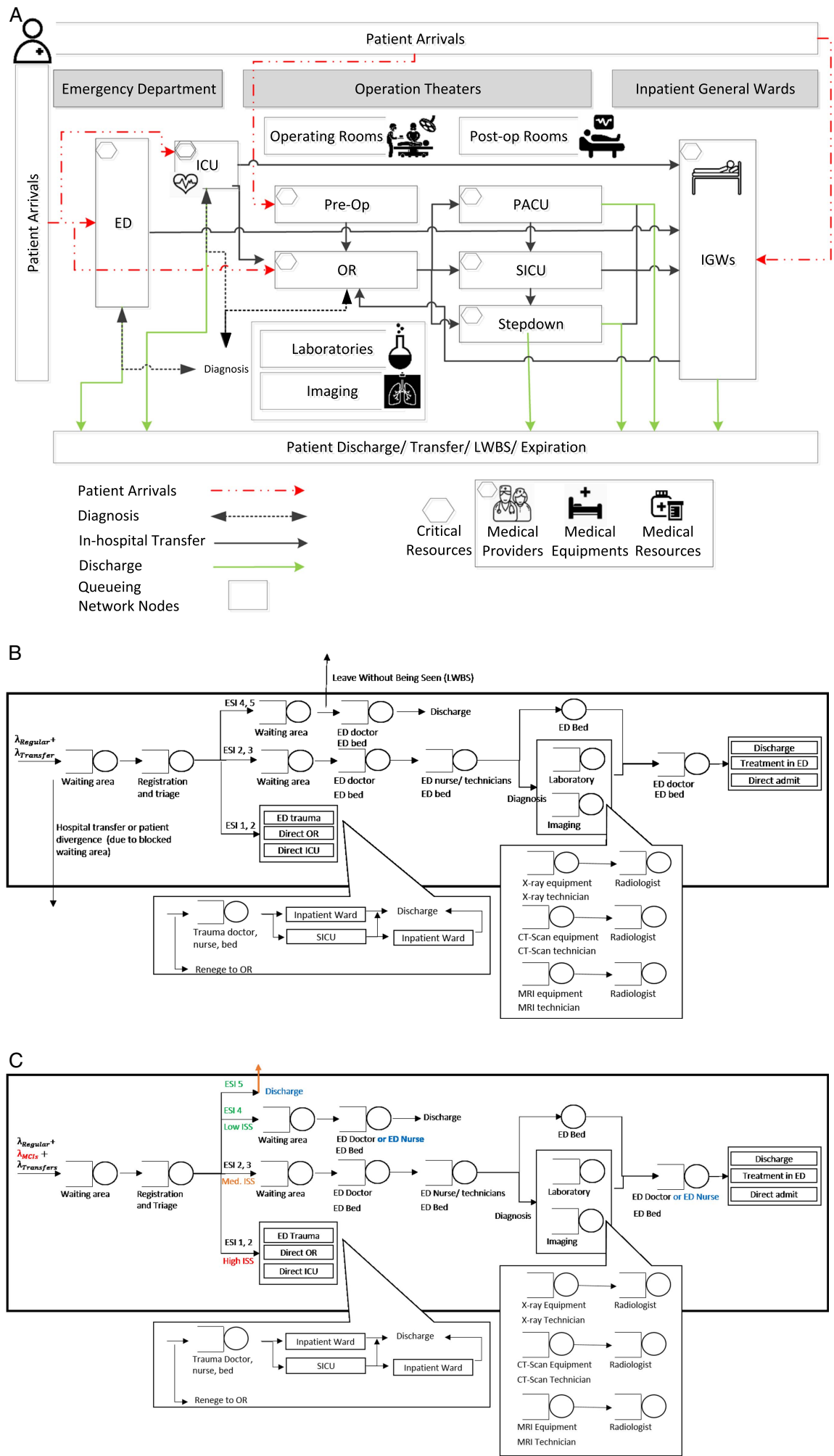
Summary of Assumptions Compared Under Routine ${ }^{10}$ and MCI Conditions

Units

ED triage

ED patient types

ED patient arrivals

ED policy and operation

ED capacity

ED service time (individual servers)

Laboratories

Preop

OR

PACU, ICU, SICU, step down

Inpatient wards

\section{Routine Conditions}

On average 15 minutes per patient

$20 \%-25 \%$ green-tagged patients

Exponential interarrivals, daily arrival mean follows bimodal normal distribution (nonstationary time-dependent mean)

ED physician visits all patients

Waiting room only

ED cots in rooms

Average 10 minutes for a visit by ED physician

TAT of 60 minutes ( $90 \%$ completion time of $<60$ minutes)

Not patients from inpatient ward

1 bed per room

Elective same-day surgical admissions on weekdays

Full expected length of stay

Taking very low risk on return of patient (low direct discharge rates)

Patient to nurse ratio of 2:1

1 or 2 cots in each room

Full expected length of stay

Patient to nurse ratio of $5: 1$

\section{Condition ${ }^{a}$}

On average 2 minutes per patient

$50 \%$ green-tagged patients ${ }^{\mathrm{b}}$

$30 \%$ yellow-tagged patients ${ }^{b}$

$20 \%$ red-tagged patients ${ }^{b}$

Exponential

Batch arrivals, dual wave phenomenon

Conditional ED admission (green-tagged patients) ${ }^{c}$

RN visits patients ${ }^{c}$

RN performs procedures ${ }^{\mathrm{C}}$

Add corridors and open spaces

Increase ED cots up to $20 \%$ of initial capacity

Average 5 minutes for a visit by ED physician

TAT of 45 minutes

Patients enter the hospital in need of operation

Additional cots can be added to each room

$90 \%$ cancellation of elective surgeries

Shorter length of stay

$10 \%$ higher direct discharge rates

Lower patient to nurse ratio (3:1)

30\% ICU capacity expansion

Add cots to rooms

Place observatory cots in corridors up to $20 \%$ of initial capacity

Shorter length of stay

$30 \%$ immediate discharge

$25 \%$ decrease in admission from ED

Lower patient to nurse ratio (10:1)

Abbreviations: ED, emergency department; ICU, intensive care unit; OR, operating room; PACU, postanesthetic care unit; RN, registered nurse; SICU, surgical intensive care unit; TAT, turnaround time.

anterview results with Johns Hopkins Hospital $(\mathrm{JHH})$ and other hospitals in the JHH system (JHHS).

buggested. ${ }^{1}$

${ }^{\mathrm{C}}$ Under state of emergency.

\begin{tabular}{|c|c|c|c|}
\hline \multicolumn{4}{|c|}{ Summary of Demand and Operational Strategies } \\
\hline Demand & $\begin{array}{c}\text { Routine }^{\mathrm{a}} \\
<200 \text { patients/day }\end{array}$ & $\begin{array}{c}\text { MCI-Pandemic } \\
\text { 250-300 patients/day }\end{array}$ & MCI I/II/III \\
\hline $\begin{array}{l}\text { Capacity Expansion } \\
\text { Strategies }\end{array}$ & $\begin{array}{l}\text { Modified Operations } \\
\text { (1) } 75 \% \text { scheduled } \\
\text { (2) Increased servic } \\
\text { (3) Early discharge } \\
\text { (4) Longer shifts for } \\
\text { (5) Opening extra a }\end{array}$ & $\begin{array}{l}\text { ns canceled } \\
\text { ts in wards } \\
\text { edical space }\end{array}$ & $\begin{array}{l}\text { Alternative Standard of Care } \\
\text { (1) Selective services } \\
\text { (2) Shorter length of stay } \\
\text { (3) Lower staff to patient ratios } \\
\text { (4) Altered patient routings }\end{array}$ \\
\hline
\end{tabular}

Abbreviation: $\mathrm{MCl}$, mass casualty incident.

aUsing highest demand, consistent with the fall flu season and $\mathrm{MCl}$ I, II, \& III, corresponding to 10, 20, and $100 \mathrm{MCl}$ patients.

\section{RESULTS AND DISCUSSION}

Results from the 7000 runs were obtained and analyzed. Reasonableness of the designed representative urban hospital and general modeling approach was assessed ${ }^{10}$ under routine conditions. Initial $\mathrm{MCI}$ model run results were discussed with emergency managers, ${ }^{11}$ and the model was further tuned to incorporate elements that were not initially identified as key. The best capacity-expansion strategies were identified. 


\section{FIGURE 3}

\section{Daily Average Number of Available Inpatient General Ward Beds in M01 and M02 in MCI-P Scenarios}

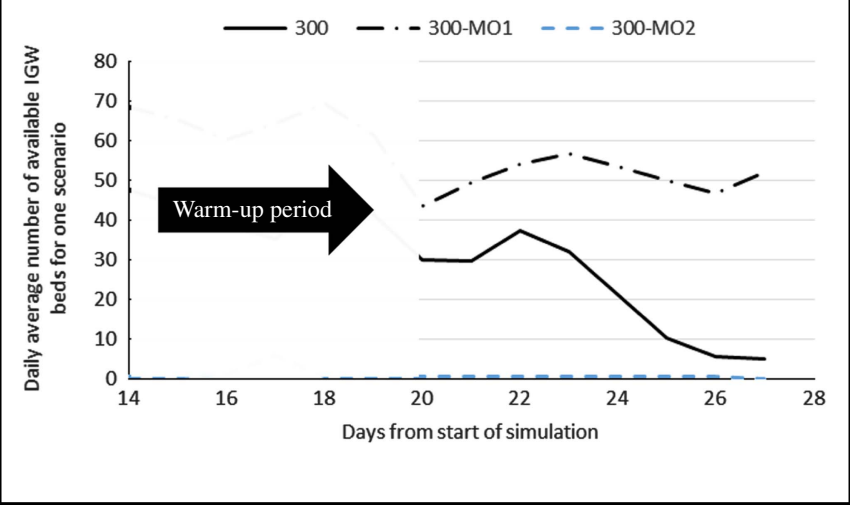

Moreover, incident time-of-day impacts and resulting dynamic effects on waiting times were investigated. This section contains results of observed queues in which significant changes were identified.

\section{Results}

A baseline was created from results of runs for a demand of 200 ED patient arrivals per day with morning and afternoon peaks with higher wait times, as is typical of similarly sized, busy urban hospitals known to operate close to their capacities. ${ }^{18}$ Incidents falling under three MCI classes are replicated:

(1) MCI-P: an increase in the total number of patients per day by up to 100 patients to replicate seasonal effects from, for example, flu season and more severe conditions of a pandemic.

(2) MCI-I and MCI-II: a one-time increase in arrivals of 10 or 20 red (critical) patients to replicate a MCI I or II, respectively; such MCIs might occur in a rail transportation accident, for example.

(3) MCI-III: a dual wave arrival on day one of $100 \mathrm{MCI}$ patients following patient types described in Table 3 and arrival pattern shown in Figure 1B for a high-casualty MCI III event.

\section{MCl-P}

The discrete event simulation model outputs are processed to obtain 7-day average patient throughput, number of transfers, and number of green patients who leave their queues without being seen (LWBS) under demand scenarios with total daily patient arrival rates of 200,250 , or 300 and routine operations and standards of care. Analysis of these outputs indicates that average waiting times are not significantly impacted by a surge in demand at this level. However, the number of transfers from the ED, number of patients diverted to another hospital upon arrival (ie, hospital refusals), and LWBS cases increased sizably.

Detailed study of waiting times along specific patient care paths revealed that the less severe patients (ESI levels 4 and 5) bare the cost of the surge through increased, often unacceptable waiting times and decreased throughput as a result of transfers. With an increase of 50 patients daily, the capacity of the waiting area for these patients was exceeded as indicated by increased waiting times at registration, high number of hospital transfers, and slight increase in LWBS cases. A sharp rise in waiting times was also noted for severely injured patients (ESI levels 1 and 2) in need of ICU or MRI equipment for diagnoses. These unmet needs greatly affect surgery theater throughput, putting patients at further risk.

Average performance improvements gained through capacityexpansion strategies (Figure 2) under the MCI-P scenario with a 100 daily patient increase are given in Table 4.

Results from Table 4 show improvements that are not necessarily proportional to the capacity changes incurred through expansion strategy implementations. Further, they indicate that an expansion strategy aimed at a particular hospital unit may have negative or positive secondary effects on other units. By example, the canceling of $75 \%$ of elective surgeries (MO1) eliminated OR waiting times and decreased postop waiting times by more than $90 \%$, but simultaneously released only $15 \%$ of the beds in the IGWs. Effects of expansion strategies transcend the units they targeted. In the experiments, speeding up processes in the ED (MO2) increased throughput in the ED itself, but simultaneously improved functionality of operation theaters and decreased bed availability in the IGWs (Figure 3). The improvement in the functionality of the operation theaters resulted from increased ED efficiency, which further decreased the probability that patients reach a critical state requiring that they be transferred to the higher acuity care paths that include operations. As patients clear the ED, more patients will be admitted to the hospital, leading to higher inpatient admission numbers and lower IGW bed availability.

As the impact of each capacity-expansion strategy extends beyond its specific target, predicting the effects across the hospital of combined strategies is difficult. For insight into the effectiveness of combining strategies, two capacity-expansion strategies were considered simultaneously under the MCI-P scenario: MO2 and ASC4. These were chosen to address bottlenecks noted along patient care paths. These modifications specifically target bottlenecks in lab test result interpretation and $\mathrm{ED}$ procedures that rely on available $\mathrm{ED}$ physicians. Each strategy alone creates marked improvements. For example, $\mathrm{MO} 2$ and ASC4 each led to an increase in throughput of ESI level 4 and level 5 patients by approximately $30 \%$ and $40 \%$, respectively. When combined, effect was a tenfold increase in throughput of the same patient categories. An added benefit is a combined effect of decreased hospital transfers and LWBS cases by roughly one-half. It is noteworthy that when implemented separately, there was no significant impact on LWBS cases and only ASC4 resulted in improvements in hospital transfers. A negative consequence, however, of this combination is that 


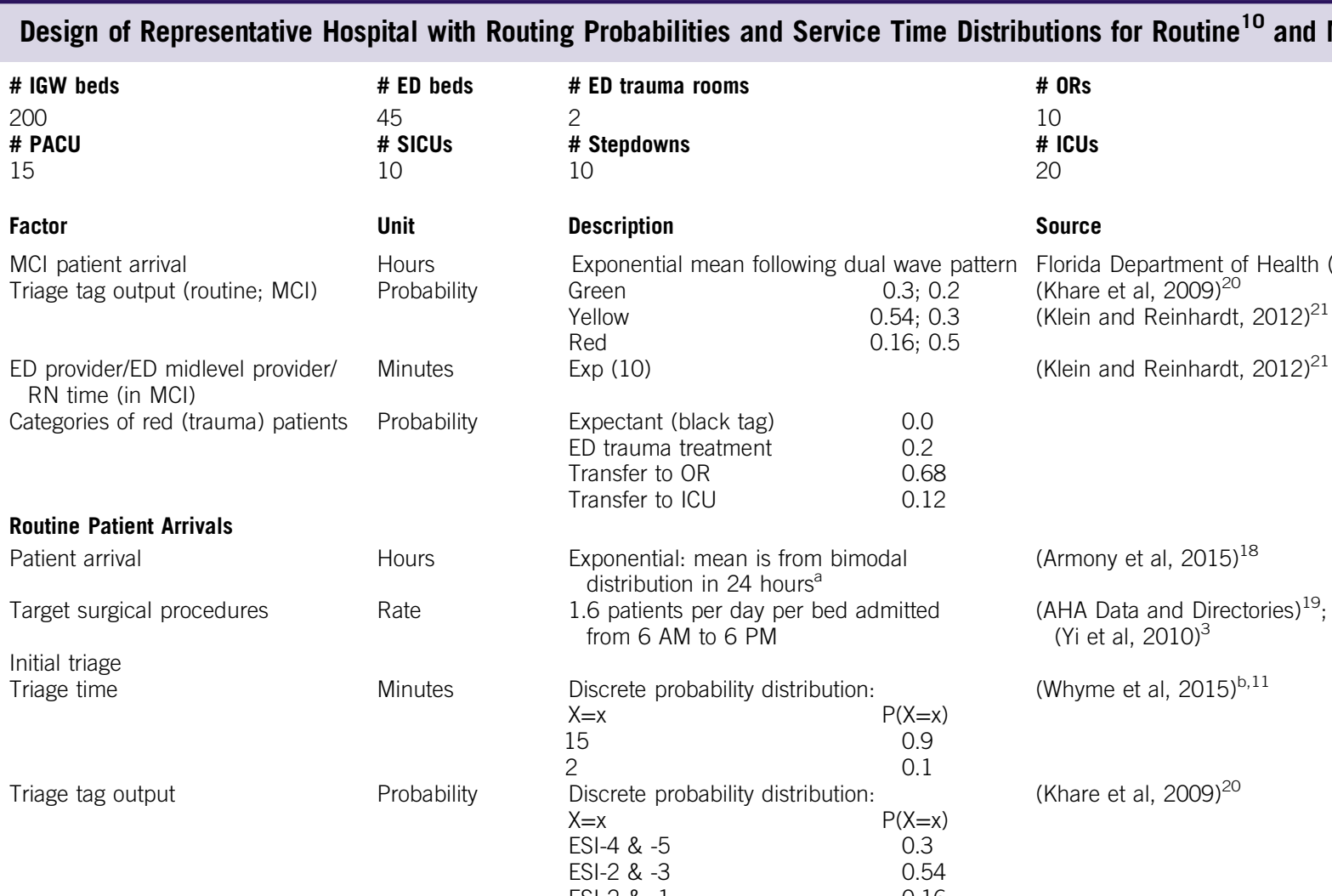

\section{ESI Level 4 and 5 Patients}

Categories

Probability

ESI-2 \& -1

Discrete probability distribution:

$\begin{array}{lr}X=x & P(X=x) \\ \text { ESI-4 } & 0.50 \\ \text { ESI-5 } & 0.50\end{array}$

$\operatorname{Exp}(10)$

(Khare et al, 2009) ${ }^{20}$

ED physician (provider)/ED midlevel Minutes provider

Leave without being seen (LWBS) Hours threshold

3, Leave w.p of 0.5

8, Leave w.p of 1

Uniform $(6,8)$

$\operatorname{Exp}(15)$

$\operatorname{Exp}(10)$

General requirements

Survival time duration

ED physician time first visit

ED nurse/technician time after first

Minutes

ED physician's visit

Diagnosis

Probability

Discrete probability distribution:

Lab test time

$X=x$

Laboratory only

Laboratory and imaging

Imaging

Radiology time (procedure)

CT scan time (procedure)

MRI time (procedure)

Radiologist time to comment on results

Treatment decision
Minutes

Minutes

Probability

Exp (60)

$\operatorname{Exp}(2)$

Discrete probability distribution:

$X=\mathrm{X}$

Radiology

CT scan

MRI

Minutes $\quad \operatorname{Exp}(15)$

Minutes

$\operatorname{Exp}(60)$

Minutes

Minutes

Probability

\section{Exp (120)}

Radiology

CT scan

MRI

Discrete probability distribution:

$\mathrm{X}=\mathrm{x}$

No treatment

ED treatment

Transfer to OR
$P(X=x)$

0.5

0.5

$P(X=x)$

0.6

0.35

0.05

(Hawkins, 2007) $^{24}$; (Jackson, 2015) ${ }^{26}$

(Whyme et al, 2016) ${ }^{11}$

(Gunn et al, 2013) ${ }^{25}$

(Gunn et al, 2013) ${ }^{25}$

(Whyme et al, 2016) $^{11}$

(Whyme et al, 2016) ${ }^{11}$

(Hurwitz et al, 2014) ${ }^{27}$

(Hawkins, 2007)24

(Whyme et al, 2016) ${ }^{11}$

(Gunn et al, 2013) ${ }^{25}$

$\operatorname{Exp}(5)$

$\operatorname{Exp}(20)$

$$
\operatorname{Exp}
$$

$P(X=x)$
\# Preops

20

(Klein \& Reinhardt, 2012) $)^{21}$

(Hsia et al, 2011) 22 ;

(Armony et al, 2015) $^{18}$

(AMEDD, 2016) ${ }^{23}$

(Khare et al, 2009) ${ }^{20}$

(Whyme et al, 2016) ${ }^{11}$

(Whyme et al, 2016) $^{11}$

0.2

0.7

0.1 


\section{TABLE 3}

\section{Continued}

\section{Factor}

ED treatment breakdown

Requires ED physician to perform

procedures

ED physician time

ED nurse

Patient monitoring time

IGW LOS ${ }^{\mathrm{d}}$

Post ED routing

Transfer delay to IGW

Discharge delay

Treatment/monitoring by nurses

ED nurse

Patient monitoring time

Post-ED routing

\section{ESI Level 1 and 2 Patients}

Categories of trauma patients

Survival time duration

ED trauma treatment

Trauma physician and nurse time

Trauma nurse time

Renege to OR

Following routing

OR

Pre OR routing

OR with preop

Preop physician time

OR physician, nurse time

Postop routing

OR mortality rate

SICU mortality rate

SICU LOS

PACU

Post PACU routing

\section{Unit}

Minutes
Minutes
Minutes
Days
Probability

Minutes

Minutes

Minutes

Minutes

Probability

Hours

Minutes

Minutes

Minutes

Probability

Probability

Minutes

Minutes

Probability

Percentage

Percentage

Days

Minutes

Probability

\section{Description}

ED procedure needed

Nurse monitoring

0.4

0.6

Exp (60)

ED physician time $+\exp (60)^{c}$

Exp (15)

Lognormal $(3.87,3.26)$

Discrete probability distribution:

$X=x$

Direct discharge

$P(X=x)$

Delayed discharge $\quad 0.47$

IGW 0.4

$\begin{array}{ll} & \\ \text { ICU } & 0.12^{\mathrm{e}}\end{array}$

Discrete probability distribution:

$X=x$

10

$P(X=x)$

30

160

Exp (40)

0.04

0.46

0.5

$\operatorname{Exp}(10)$

$\operatorname{Exp}(60)$

Discrete probability distribution:

$\begin{array}{lr}X=x & P(X=x) \\ \text { IGW } & 0.25 \\ \text { Discharge } & 0.75\end{array}$

Discharge

0.75

Discrete probability distribution:

$X=x$

$P(X=X)$

ED trauma treatment

0.2

Transfer to OR

0.68

Transfer to ICU

Uniform $(0.5,1.5)$

Max (Exp [45], ED lab/ED lab and imaging test time duration)

Max (Exp [45], ED lab/ED lab and radiology test time duration $)^{\mathrm{g}}$

15
Discrete probability distribution:

$X=\mathrm{x}$

w.p. 1

IGW

$P(X=x)$

Postops

0.8

Preop required

Preop not required

$0.01^{\text {h }}$

0.99

$\operatorname{Exp}(30)$

$\operatorname{Exp}(90)^{i}$

Discrete probability distribution:

$X=\mathrm{X}$

SICU

PACU

Stepdown

National average over all

patient types

Range 8\%-19\% used

Triangle distribution $(0.25,6,2)$

$P(X=x)$

0.3

0.4

0.3

2.9

13.5

Normal distribution $(95,43)$

Discrete probability distribution:

$\begin{array}{lc}X=x & P(X=x) \\ \text { IGW } & 0.4 \\ \text { Stepdown } & 0.3 \\ \text { Discharge }^{k} & 0.3\end{array}$

\section{Source}

(Whyme et al, 2016) ${ }^{11}$; Hurwitz et al, $2014)^{27}$

(Whyme et al, 2016) $^{11}$

(Whyme et al, 2016) ${ }^{11}$

(Whyme et al, 2016) $^{11}$

(Armony et al, 2015) $^{18}$; (AHA, 2016) ${ }^{19}$

$(\text { AHA, 2016) })^{19}$; (Shi et al, 2015) $)^{28}$

(Armony et al, 2015) ${ }^{18}$

(Whyme et al, 2016) $^{11}$

$\left(\right.$ AHA, 2016) ${ }^{19}$

$(\mathrm{AHA}, 2016)^{19}$

$(\mathrm{AHA}, 2016)^{19}$; (Shi et al, 2015) ${ }^{28}$;

(Khare et al, 2009)

$(\mathrm{AHA}, 2016)^{19}$

$(\mathrm{AHA}, 2016)^{19}$

$(\mathrm{AHA}, 2016)^{19}$

$(\mathrm{AHA}, 2016)^{19}$

$(\mathrm{AHA}, 2016)^{19}$

(Barbagallo et al, 2015) ${ }^{29}$

(Barbagallo et al, 2015) ${ }^{29}$; (AHA, 2016) $)^{19}$

(Whyme et al, 2015) $^{11}$; (AHA, 2016) ${ }^{19}$

(Armony et al, 2015) $^{18}$;

(QuickStats, 2016) 30

(QuickStats, 2016) $^{30}$

(SCCM, 2016)

(Waddle et al, 1998) (22; $^{2}$

(Barbagallo et al, 2015) ${ }^{29}$

(AHA, 2016) ${ }^{19}$; (Whyme et al,

2016) ${ }^{11}$; (Khare et al, 2009) ${ }^{20}$ 


\section{TABLE 3}

\section{Continued}

\section{Factor}

Stepdown LoS (for patients

transferred in from PACU)

Post stepdown routing

Post stepdown routing

Stepdown LoS (for patients

transferred in from OR)

IGW LoS

ICU LOS

ICU mortality rate

$\begin{array}{llc}\begin{array}{l}\text { Unit } \\ \text { Days }\end{array} & \begin{array}{l}\text { Description } \\ \text { Triangle distribution }(0.5,1.5,1)\end{array} \\ \text { Probability } & \text { Discrete probability distribution: } \\ & & \\ & \mathrm{X}=\mathrm{x} & \mathrm{P}(\mathrm{X}=\mathrm{x}) \\ \text { Probability } & \mathrm{IGW} & 0.5 \\ & \text { Discharge } & 0.5 \\ \text { Days } & \text { Triangle distribution }(0.25,6,2) & \\ & & \\ \text { Days } & \text { Normal distribution }(4.5,2) & \\ \text { Days } & \text { Triangle distribution }(0.25,6,2) & \\ \text { Percentage } & \text { Range } 10 \%-29 \% \text { used 20\% } & \end{array}$

\section{Source}

(SCCM, 2016) $)^{31}$

(Whyme et al, 2016) $^{11}$;

(Armony et al, 2015) $^{18}$

$(\mathrm{SCCM}, 2016)^{31}$

$(\mathrm{AHA}, 2016)^{19} ;(\text { Armony et al, 2015) })^{18}$

$(\mathrm{SCCM}, 2016)^{31}$

(QuickStats, 2016) (30 $^{30}$

Abbreviations: ED, emergency department; ICU, intensive care unit; IGW, LoS, length of stay; inpatient general ward; MCI, mass casualty incident; OR, operating room; PACU, postanesthetic care unit; preop, preoperation room; postop, postoperation room; RN, registered nurse; SICU, surgical intensive care unit; TAT, turnaround time.

aSample of patient arrival realization. ${ }^{10}$

bInterview results with JHHS.

${ }^{\mathrm{c} A d d i t i o n a l}$ ED nurse time is for patient monitoring and transfer preparation.

${ }^{\mathrm{I}} \mathrm{CU}$ and inpatient length of stay are shown to be significantly different between patients in various ISS levels.

'We assumed that $12 \%$ of all ED and elective admissions are directed to the ICU. This assumption was based on large-scale studies of ICU utilization and stakeholder feedback.

fInpatient admission rate is 57\% for ESI level 2 and $31 \%$ for ESI level 3 patients. Remainder are discharged.

gNurse remains in charge until patient are released to the next unit or discharged.

${ }^{\mathrm{h}} \mathrm{OR}$ preparation for ED transferred patients is completed in ED and during transfer.

iNot including turnaround time.

jMortality rate can be significant, such as $4.9 \%$ for cardiovascular patients, or as low as $0.92 \%$ for orthopedic surgeries. ${ }^{33}$

kPACU-discharge patient path corresponds to same-day/outpatient surgery. Patients in PACU-stepdown and OR-stepdown paths that are released less than 24 hours after surgery contribute to about $60 \%$ of OR patients as estimated for 2013 US community hospitals. ${ }^{19}$

it led to an increase in waiting time for the OR by blocking the IGWs and postop units. It might be more effective and less deleterious to add staffed beds to the IGWs. Other examples of superadditive impacts of combined strategies were observed and included in Appendix B.

\section{$\mathrm{MCl}-\mathrm{I}$ and $\mathrm{MCl}-\mathrm{II}$}

To analyze the impact of a MCI I or MCI II on hospital performance under routine operations and standards of care, 2-day performance measure averages were recorded. From these results it was noted that, while the additional 10 critical patients did not affect overall ED performance, all needed surgery, and thus waiting times in the ORs increased from less than 5 minutes to 100 minutes. It is recommended in military triage guidelines that waiting times for this class of patients not exceed 90 minutes. The model thus will choose to transfer the patients whose expected waiting times exceed this limit. Consequently, in the MCI-II case, no further increase in waiting times at the ORs was noted. Additional performance loss was observed in the postop units, which suffered from a doubling of waiting times and an increase of 300 minutes in average transfer times from the $\mathrm{OR}$ to the stepdown unit and an increase of 417 minutes in average transfer times from the PACU to the stepdown unit. The important role of well-functioning trauma rooms in stabilizing critical patients was also noticeable.

For MCI-I or MCI-II, capacity-expansion strategy MO1 (canceling elective surgeries) was found from the experiments to provide the needed OR capacity to handle the extra critical patients for these incident scenarios. A less intrusive version of $\mathrm{MO}$, in which surgery patients who arrived at the hospital prior to the MCI are moved from preop to the IGWs so that their surgeries can be delayed until after the $\mathrm{MCI}$ patients are served, was tested. This adapted MO1 strategy eliminated $\mathrm{OR}$ waiting times and reduced average waiting times in postop units; the average SICU, PACU, and stepdown waiting times dropped by $62 \%, 60 \%$, and $42 \%$, respectively.

\section{$\mathrm{MCl}-\mathrm{III}$}

Results for 100 additional MCI patient arrivals to the hospital following the dual wave phenomenon (explained in Methods) under routine operations and standards of care indicate an untenable situation. The main entry point to the hospital for all patient types, including those arriving by ambulance, quickly became blocked, patients were diverted, and LWBS cases rose sharply. There was also an increase in ED waiting times. Surprisingly, however, the waiting times in the operation theaters was not as great as they were for the MCI-I and MCI-II scenarios, perhaps due to the dual-wave arrival pattern in which the critical patients arrivals are more spaced out over time.

ASC3 and MO1 capacity-expansion strategies can be combined to increase ED capacity. The results of runs aimed at testing this hypothesis showed an acceptable increase in waiting times in the ED for a significant improvement or even elimination of waiting times for the ORs, SICU, and stepdown unit. 
TABLE 4

7-Day Average Performance Improvements Under Capacity Expansion Strategies for MCI-P

\author{
Capacity \\ Expansion \\ Strategies \\ MO1 $\quad 75 \%$ cancellation of \\ elective surgeries/ \\ operations \\ MO2 \\ Speed up ED processes \\ by $25 \%$

\section{Waiting Times} \\ No wait time in $\mathrm{OR}$ \\ $90 \%$ decrease in SICU \\ $50 \%$ decrease in stepdown \\ No wait time in OR \\ 95\% decrease in SICU and PACU \\ $70 \%$ decrease in stepdown \\ $(\mathrm{N})^{\mathrm{a}}$ blocked IGWs
}

MO3

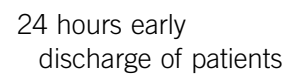
discharge of patients in IGW

MO4

Longer shifts for staff: ED doctors and stepdown unit

MO5
$50 \%$ increase in waiting areas and $30 \%$ increase in ED beds

ASC 1 Direct exit of $50 \%$ of ESI 4/5 patients after initial triage point

ASC 2 1-Day early discharge in stepdown units and IGWs

\section{ASC 3 Lower staff-to-patient ratio in post operation units and IGWs Adding $25 \%$ cots and beds in operation theaters and IGW}

ASC 4
$5 \%$ decrease in ED for ESI 2/3 patients

$5 \%$ decrease in postop care

$50 \%$ decrease for patients in no need

of surgery and ICUs

Increase of IGW wait time from 2 to

6 hours for patients with lowest priorities

Operating theaters blocked:

11 hour wait for ICU

50 hour wait for ORs

(N) blocked IGWs

$75 \%$ decrease in postop units

Slight decrease in waiting times in ED after triage (average of 5 minutes)

$47 \%$ in registration

(N) $53 \%$ increase in ED waiting room for

ESI 3, 4, \& 5

Average of $40 \%$ to $50 \%$ decrease in postop units

No wait time in OR

$50 \%$ decrease in preop

Decrease in postops:

$92 \%$ for SICU

$40 \%$ for Stepdown

$65 \%$ for PACU

95 decrease in ED waiting time for ESI 2/3 patients, $97 \%$ decrease in waiting for ED physician to perform a procedure or confirm a diagnosis $94 \%$ decrease in wait time of postop units (N) blocked IGWs

\section{Patient Throughput}

Opening up 15\% IGW capacity that can increase the floor admission

$34 \%$ increase of ESI $4 / 5$ (treated in ED)

$23 \%$ increase in ESI 2/3 (for patients that are receiving full treatment in ED)

$27 \%$ increase in floor admission $33 \%$ increase in ESI 1 (ED-ICU)

Opening up 30\% IGW capacity that can increase the floor admission $10 \%$ increase of patient throughput in operation theaters

$30 \%$ increase of ESI $4 / 5$

$20 \%$ increase in ESI 2/3

No significant change

No significant change for other groups

$22 \%$ increase in stepdown units when transfer directly from OR

$19 \%$ increase in stepdown $7 \%$ increase in SICU

\section{Patient Transfers}

No significant changes in total

No significant changes in total

No significant changes in total

$30 \%$ decrease in hospital transfer

$52 \%$ decrease of hospital transfers

(N) $88 \%$ increase of ED main treatment transfers and expirations

$10 \%$ decrease in LWBS

$16 \%$ decrease in hospital transfers

(N) $28 \%$ increase in LWBS

No significant changes in total 9-fold increase of ESI 4/5 patient throughputs

$23 \%$ increase in ED throughput of ESI 2/3 patients without imposing waiting time on other units
$49 \%$ decrease in hospital transfers $26 \%$ decrease in ED main treatment transfers

Abbreviations: ED, emergency department; ESI, Emergency Severity Index; ICU, intensive care unit; IGW, inpatient general ward; LWBS, leave without being seen; $\mathrm{MCl}$, mass casualty incident; $\mathrm{MCl}-\mathrm{P}$, an increase in the total number of patients per day by up to 100 patients to replicate seasonal effects from, for example, flu season and more severe conditions of a pandemic; OR, operating room; PACU, postanesthetic care unit; preop, preoperation room; postop, postoperation room; RN, registered nurse; SICU, surgical intensive care unit.

${ }^{a}(\mathrm{~N})$ indicates statistically significant negative impact. 
FIGURE 4

\section{Hospital Functionality Over 24 Hours of the Transient Period (Day 1 Following the MCI III). Abbreviations: ED, emergency department; ESI, Emergency Severity Index; $\mathrm{MCl}$, mass casualty incident.}

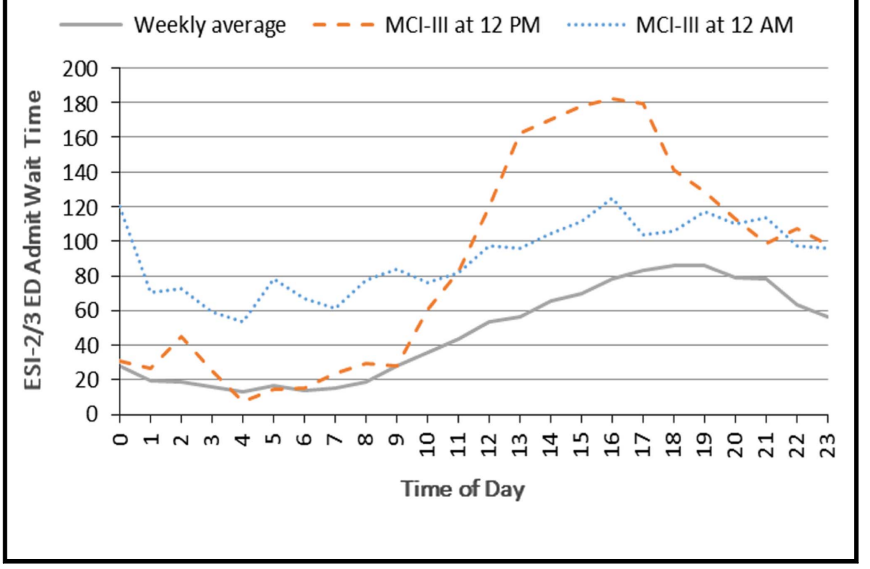

\section{Timing and Dynamics}

Experimental results discussed thus far assumed that the first incident-related arrival would occur at noon. Additional runs under both MCI-II and MCI-III scenarios were conducted with an incident occurrence near midnight. Overall, nighttime waiting times for the ORs were comparatively low as there are no scheduled operations, and patient processing times were generally higher. Results in Figure 4 also indicate that an incident arising at noon would lead to higher peak waiting times, despite faster patient processing. Consequently, the average mortality rate is expected to be greater ( 2.7 versus 0.67 patients per day) for an incident arising at noon as opposed to midnight.

\section{CONCLUSIONS}

Several important observations were made from analysis of the numerical run results and proposed capacity-expansion strategies. First, response capability depends on not only the number of critical patients but their arrival pattern and injury types. A hospital can better serve a larger number of critical patients if they arrive over time than it can fewer critical patients arriving all at once. Such insights can be used in regional response planning and can help a hospital in choosing between potential capacity enhancement options depending on its trauma response capability. For example, for a trauma level I hospital it might be best to increase the number of IGW beds to free up space in the ED and ORs, whereas for a trauma level III hospital, enhancing the capacity of the ED directly could better enhance response. Additionally, the modeling approach proposed here reveals that capacity-expansion strategies, such as increasing the number of ED beds, may not be effective without concomitant enhancements to other facilities or increases in indirectly related resources. Second, the results show that capacity enhancement plans, when combined, can have a superlinear impact if paired carefully. In fact, only 2 to 3 enhancement types were needed to reach acceptable service levels for suitable combinations.

A third observation, a critical point for hospital bottleneck analysis, is that the longest wait times were not necessarily found at bottlenecks, but often were noted at the entry to downstream services. Analysis steps taken in this work can be replicated to find the initial point of bottlenecks in hospital patient flows. Fourth, the model run results indicate that implementing strategies of alternative standards of care was more effective in improving hospital performance than implementing strategies that would modify operations. Allowing nurses to handle low-injury class patients and early discharge strategies were found to be the most effective of such changes in standards. The effects of such strategies on long-term patient wellness (ie, 30-day return risk) needs further study. Finally, and more generally, the results show the criticality of taking a holistic view of hospital functionality in terms of both interactions along patient care paths that cross units and medical staff and resource availability and constraints.

The accuracy with which the model replicates a specific hospital, and thus estimates its performance, is a function of how well it simulates actual conditions in terms of not only demand, space, physical resources, and processes, but actions and decisions of the hospital personnel. To calibrate the model accordingly will require observation of the hospital and its functionality under rare events.

This work highlights minimum required information to monitor hospital functionality and captures the dependencies between those elements in MCI conditions that would help hospitals with different sizes to replicate and rebuild the model. The proposed modeling framework could also serve within a real-time forecasting tool for predicting next period resource needs and capacity requirements. Predictions can be employed to choose appropriate capacity enhancement modifications, changing priorities, or demand management strategies (eg, redirecting patients) and forecast their effectiveness as compared to the costs and difficulties associated with their implementation.

\section{About the Authors}

George Mason University, Fairfax, Virginia (Drs TariVerdi, Miller-Hooks); and National Center for Disaster Medicine and Public Health, Uniformed Services University of the Health Sciences, Bethesda, Maryland (Dr Kirsch).

Correspondence and reprint requests to Dr Elise Miller-Hooks, George Mason University, 4400 University Drive, Fairfax, VA 22030 (e-mail: miller@gmu. edu). Phone: 703.993 .1685

\section{Funding}

This work was funded by the National Science Foundation. This support is gratefully acknowledged, but implies no endorsement of the findings. 


\section{SUPPLEMENTARY MATERIAL}

To view supplementary material for this article, please visit https://doi.org/10.1017/dmp.2018.4

\section{REFERENCES}

1. Florida Department of Health website. http://www.floridahealth.gov/. Accessed April 14, 2017.

2. Hick JL, Barbera JA, Kelen GD. Refining surge capacity: conventional, contingency, and crisis capacity. Disaster Med Public Health Prep. 2009;3 (suppl 1):S59-67.

3. Yi P, George SK, Paul JA, et al. Hospital capacity planning for disaster emergency management. Socioecon Plann Sci. 2010;44:151-160.

4. Jacobson EU, Argon NT, Ziya S. Priority assignment in emergency response. Oper Res. 2012;60:813-832.

5. Comfort LK. Coordination in rapidly evolving disaster response systems: the role of information. Am Behav Sci. 2004:48:295-313.

6. Malavisi M, Cimellaro GP, Terzic V, et al. Hospital emergency response network for mass casualty incidents. Am Soc Civ Eng. 2015:1573-1584. http:// ascelibrary.org/doi/10.1061/9780784479117.135. Accessed January 3, 2017.

7. Aylwin CJ, König TC, Brennan NW, et al. Reduction in critical mortality in urban mass casualty incidents: analysis of triage, surge, and resource use after the London bombings on July 7, 2005. Lancet Lond Engl. 2006;368:2219-2225.

8. Lin L, Cochran JK. Metamodels of production line transient behaviour for sudden machine breakdowns. Int J Prod Res. 1990;28:1791-1806.

9. Stolletz R, Lagershausen S. Time-dependent performance evaluation for loss-waiting queues with arbitrary distributions. Int J Prod Res. 2013;51:1366-1378.

10. TariVerdi M, Miller-Hooks E, Kirsch TD, et al. A Resource-Constrained, Whole-Hospital Modeling Framework for Evaluating Operational Strategies Under Routine and Surge Demand Scenarios. In review.

11. Whyme D, Notobartolo C, Sasenick D, et al. Multiple interviews on hospital design, operation, and resource management. 2015.

12. Emergency Severity Index Algorithm. http://www.esitriage.org/algo rithm.asp. Accessed December 19, 2016.

13. Critical Illness \& Trauma Foundation, Inc., Bozeman, Montana. http:// citmt.org/index2.htm. Published 2016. Accessed January 24, 2017.

14. Paul JA, George SK, Yi P, et al. Transient modeling in simulation of hospital operations for emergency response. Prehosp Disaster Med. 2006;21:223-236

15. Durkins ME. Fatalities, nonfatal injuries, and medical aspects of the Northridge Earthquake. In Woods MC, Seiple WR, eds. The Northridge, California Earthquake of 17 January 1994. Sacramento, CA: California Department of Conservation, Division of Mines and Geology; Special Publication 1996:187-213.

16. Natural disasters and severe weather. Center for Disease Control and Prevention website. https://www.cdc.gov/disasters/index.html. Published 2016. Accessed January 24, 2017.

17. Kelen GD, McCarthy ML, Kraus CK, et al. Creation of surge capacity by early discharge of hospitalized patients at low risk for untoward events. Disaster Med Public Health Prep. 2009;3:S10-S16.
18. Armony M, Israelit S, Mandelbaum A, et al. On patient flow in hospitals: a data-based queueing-science perspective. Stoch Syst. 2015;5:146-194.

19. AHA data and directories. American Hospital Association website. http://www.aha.org/research/rc/stat-studies/data-and-directories.shtml. Accessed December 22, 2016.

20. Khare RK, Powell ES, Reinhardt G, et al. Adding more beds to the emergency department or reducing admitted patient boarding times: which has a more significant influence on emergency department congestion? Ann Emerg Med. 2009;53:575-585.

21. Klein MG, Reinhardt G. Emergency department patient flow simulations using spreadsheets. Simul Healthc 2012;7:40-47.

22. Hsia RY, Asch SM, Weiss RE, et al. Hospital determinants of emergency department left without being seen rates. Ann Emerg Med. 2011;58: 24-32.e3.

23. US Army Medical Department Center \& School Portal. http://www.cs. amedd.army.mil/borden/Portlet.aspx? $\mathrm{ID}=\mathrm{cb} 88853 \mathrm{~d}-5 \mathrm{~b} 33-4 \mathrm{~b} 3 \mathrm{f}-968 \mathrm{c}$ 2cd95f7b7809. Accessed December 19, 2016.

24. Hawkins RC. Laboratory turnaround time. Clin Biochem Rev. 2007; 28:179-194.

25. Gunn AJ, Mangano MD, Pugmire BS, et al. Toward improved radiology reporting practices in the emergency department: a survey of emergency department physicians. J Radiol Radiat Ther. 2013;1(2):1013.

26. Jackson WL. In radiology, turnaround time is king. Diagnostic Imaging website. http://www.diagnosticimaging.com/practice-management/radiol ogy-turnaround-time-king. Published 2015. Accessed December 22, 2016.

27. Hurwitz JE, Lee JA, Lopiano KK, et al. A flexible simulation platform to quantify and manage emergency department crowding. BMC Med Inform Decis Mak. 2014;14:50.

28. Shi P, Chou MC, Dai JG, et al. Models and insights for hospital inpatient operations: time-dependent ED boarding time. Manag Sci. 2015;62:1-28.

29. Barbagallo S, Corradi L, de Ville de Goyet J, et al. Optimization and planning of operating theatre activities: an original definition of pathways and process modeling. BMC Med Inform Decis Mak. 2015;15:38.

30. QuickStats: Death Rate* From Complications of Medical and Surgical Care Among Adults Aged $\geq 45$ Years, by Age Group - United States, 1999-2009. US Center for Disease Control website. https://www.cdc. gov/mmwr/preview/mmwrhtml/mm6137a6.htm. Published 2012. Accessed December 22, 2016.

31. Research/Quality. Society of Critical Care Medicine website. http:// www.sccm.org/Research/Pages/default.aspx. Accessed December 22, 2016.

32. Waddle JP, Evers AS, Piccirillo JF. Postanesthesia care unit length of stay: quantifying and assessing dependent factors. Anesth Analg. 1998;87:628-633.

33. Bhattacharjee P, Ray PK. Patient flow modelling and performance analysis of healthcare delivery processes in hospitals: a review and reflections. Comput Ind Eng. 2014;78:299-312.

34. Kirchheimer B. Full house: after years of decline, inpatient admissions are rising, pushing new construction. Mod Healthc. 2001; $31: 28-31$. 